Anuario Latinoamericano Ciencias Políticas

y Relaciones Internacionales

vol. 8, 2019

pp. $155-171$

\section{De presidentes y ministros. Cuestión de género en los casos de Argentina, Brasil y Chile}

\author{
Of Presidents and Ministers. Gender Issue \\ in the Cases of Argentina, Brazil and Chile
}

\author{
María Emilia Perri* \\ INSTITUTO DE HUMANIDADES Y CIENCIAS SOCIALES DEL LITORAL \\ CONSEJO NACIONAL DE INVESTIGACIONES CIENTÍFICAS Y TÉCNICAS \\ UNIVERSIDAD NACIONAL DEL LITORAL, SANTA FE, ARGENTINA \\ $\triangle$ maemilia.perri@gmail.com \\ https://orcid.org/0000-0003-4316-5633
}

\section{RESUMEN}

En las últimas décadas la mujer ha ganado espacios antes impensados, transformándose en un actor importante tanto en lo público como en lo privado. América del Sur fue testigo de la llegada de mujeres a las presidencias de Chile, Brasil y Argentina, todas a través del mandato popular. Hoy dichas mujeres ya no están en el poder. Es necesario preguntarse ¿Cómo impactó ello en la configuración de los ministerios desde una perspectiva de género? Con este trabajo se buscó identificar la incidencia de la llegada de las mujeres a la presidencia sobre la composición de las carteras ministeriales en clave de género. ¿La llegada de una mujer a la presidencia asegura la apertura de espacios para el colectivo femenino?

PALABRAS CLAVE: carteras ministeriales, presidentas, género, América Latina.

\begin{abstract}
In recent decades, women have gained previously unthinkable spaces, becoming an important actor both in public and private sphere. South America has witnessed the arrival of women to the presidency of Chile, Brazil and Argentina, all through the popular mandate. Today these women are no longer in power. But it is necessary to ask: How did it impact the power configuration from a gender perspective? The purpose of this work was to identify the incidence of the arrival of women in the presidency on the composition of ministerial portfolios in terms of gender. Does the arrival of a woman to the presidency ensure the opening of spaces for the female collective?
\end{abstract}

KEYWORDS: ministerial portfolios, women presidents, gender, Latin America.

* Becaria Interna Doctoral Consejo Nacional de Investigaciones Científicas y Técnicas Universidad Nacional del Litoral. Doctoranda en Ciencia Política (UNL).
DOI: 10.17951/al.2019.8.155-171 
Dossier

América Latina: género y política

\section{Introducción}

Cuando uno observa las sociedades latinoamericanas puede ver que la mujer ocupa el $50 \%$ de ellas. Incluso a lo largo de las últimas décadas ha comenzado a ocupar y a exigir espacios que antes le eran prohibidos. El ámbito político era uno de ellos, y, sin embargo, en América del Sur, en los últimos 10 años, hemos sido testigos de la llegada de mujeres a las presidencias nacionales, todas a través del mandato popular (ejemplo de ello son Argentina, Brasil y Chile). ¿Genera esto nuevos espacios para las mujeres? ¿Amplía los ya existentes? Diversas expresiones públicas y análisis políticos hablan de que la llegada de la mujer a los cargos ejecutivos nacionales significa un cambio de paradigma respecto de su participación política.

Con este trabajo se busca mostrar, a través de un análisis cuantitativo, si la incorporación de mujeres presidentas ayuda al empoderamiento de la mujer y si modifica las estructuras políticas dadas. Para ello se tomarán como casos comparativos a Argentina, Brasil y Chile, dado que en estos tres países las mujeres han logrado llegar al cargo público electivo de mayor jerarquía.

Primero, será necesario hacer un repaso sobre la situación institucional de cada país, para luego observar, a partir de la bibliografía actual, cómo afecta el género del presidente a la composición de su gabinete ministerial. Para realizar el análisis, se observarán las características generales de los tres países y las herramientas con las que cuentan para pensar la apertura del espacio político ejecutivo a la mujer.

En función de todo lo anterior se establece como hipótesis guía que la incorporación de mujeres presidentas aumenta la presencia femenina en carteras ministeriales, incluso en las denominadas "de alto prestigio". Se tomará esta hipótesis como marco para realizar el análisis comparativo de los casos de Argentina, Brasil y Chile, desde el retorno a la democracia de cada país hasta el año 2018; lo que nos permitirá establecer en qué contexto se da una mayor y mejor inclusión de la mujer en cargos ejecutivos y carteras ministeriales a nivel nacional. La variable independiente será la presidencia femenina, mientras que la variable dependiente utilizada será el número de mujeres ministras. La elección de los casos se sustenta sobre similitudes que los tres países comparten respecto a su historia institucional y a la proximidad territorial, ya que esto podría señalar procesos similares en cuanto a ampliación de derechos políticos de mujeres.

Para llevar adelante dicho análisis, se dividirá el trabajo en tres partes. La primera intentará explicar las diferencias y similitudes entre los sistemas políticos de los tres países, a la luz de la bibliografía respecto del tema. Luego, se abordará la participación de mujeres a lo largo del tiempo en los poderes ejecutivos nacionales. Y finalmente, se esbozarán las conclusiones obtenidas al respecto. Este estudio busca ser un puntapié inicial para pensar, a partir de datos de la realidad, el rol que ocupan las mujeres en la política latinoamericana y reflexionar si su mera participación modifica estructuras de poder existentes. 


\section{Sistemas latinoamericanos y mujeres}

El siglo XX ha sido el siglo de ampliaciones de derechos para las mujeres, del voto y de la representación femenina. Como hito de las últimas décadas del siglo pasado, podemos señalar las recomendaciones de la Organización de las Naciones Unidas. En la Convención sobre la Eliminación de Todas las Formas de Discriminación contra las Mujeres y la Plataforma de Acción de la Conferencia Mundial de Beijing (CEDAW, por sus siglas en inglés) se establecieron tres tipos de estrategias institucionales para la promoción de las mujeres en los procesos de decisión política (Archenti y Tula, 2008): planes y programas de igualdad y trato; oficinas mujer en los ejecutivos; cuotas de género.

Estas recomendaciones establecen que se debe poner en discusión todos los ámbitos políticos en clave de género y pensar estrategias particulares para cada uno de ellos, según el contexto social y cultural. Si bien esto hace pensar en la heterogeneidad que presentan las distintas sociedades, es necesario remarcar que, de todas las desigualdades, la de género está siempre presente. La desigualdad de género trasciende la heterogeneidad antes marcada (Ruiz Seisdedos y Grande Gascón, 2015).

Los países aquí analizados han incluido diversas combinaciones de dichas estrategias. La mixtura entre ellas debería mostrar un aumento de la participación femenina en todos los ámbitos políticos. En este trabajo nos concentraremos en lo sucedido dentro del ámbito ejecutivo, observando cómo los gobiernos han incorporado a mujeres a la cabeza de carteras ministeriales y el rol que las mujeres presidentas han tenido en ello ${ }^{1}$.

La especificidad del poder ejecutivo en sistemas presidencialistas debe ser remarcada para la mejor comprensión del objeto de estudio aquí planteado. En primer lugar, el cargo implica una unipersonalidad que conlleva a que en una misma figura se concentre la jefatura de gobierno y de Estado. A su vez, esto deriva en la claridad que supone la línea de mando: "La conformación del gabinete depende pura y exclusivamente de él, quien designa y remueve a sus ministros. Estos no pueden integrar el parlamento simultáneamente." (Pegoraro y Zulcovsky, 2006, p. 146).

En este sentido, podríamos pensar que la orientación ideológica del presidente podría ser un factor importante a la hora de la inclusión de mujeres ministras, suponiendo que los partidos de izquierda son más abiertos a la inclusión de colectivos minoritarios (Baldez, 2012; Htun, 2005). Diversos estudios establecen que en América Latina no se observan grandes diferencias en este sentido, todos los partidos buscan el apoyo de mujeres y las postulan en determinados espacios, con lo cual la ideología no sería una variable importante en la conformación del gabinete (Escobar-Lemmon, Schwindt-Bayer y Taylor-Robinson, 2012; Poskocilova, 2015).

1 Para interiorizarse más respecto de lo sucedido en Latinoamérica en los niveles legislativos o judiciales se recomienda leer los trabajos de Archenti y Tula $(2008,2014)$, Llanos y Sample (2008), Marx, Borner y Caminotti (2007).
De presidentes y ministros. Cuestión de género en los casos de Argentina, Brasil y Chile

María Emilia Perri 
Dossier América Latina: género y política
Así pues, es necesario analizar la variable de los estereotipos de género a la hora de la conformación de los gabinetes ministeriales. Según diversos trabajos, el espacio ejecutivo suele ser pensando desde lógicas patriarcales, distinguiendo maneras masculinas y femeninas de ejercer el poder. Nos sostendremos sobre aquellos estudios que cuestionan si el aumento de la participación de mujeres dentro del poder ejecutivo se debe a la aparición de Jefas de Estado (Seisdedos y Grande Gascón, 2015). Estos estudios establecen que las mujeres lideran y ejercen el poder de manera cualitativamente diferente. De ser así, podríamos observarlo en la manera en que conforman sus ministerios, con una mayor perspectiva de género.

En cuanto a los ámbitos ministeriales, hay una amplia bibliografía que específica las características generales de estos ámbitos y las dificultades que se les presentan a las mujeres en cuanto al acceso a los mismos. El principal valor que se sostiene es que los gabinetes deberían parecerse más a la población que representan (Borrelli, 2002).

Al observar hacia dentro de los ministerios, se plantea la existencia de jerarquías a la hora de hablar de ellos, y cómo esto se sustenta sobre estereotipos de género. Tomaremos la distinción de ministerios que realizan Escobar-Lemmon y Taylor-Robinson $(2005,2009)$ para hacer referencia a la importancia política de los gabinetes. Se establece que los ministerios pueden ser divididos entre gabinetes de "bajo prestigio", de "mediano prestigio" y de "alto prestigio", lo cual es determinado por el control que tienen sobre las políticas y los recursos. Entre los ministerios de bajo prestigio se encuentran los de niñez y familia, cultura, ciencia y tecnología, deporte, turismo asuntos de mujer, reforma del Estado y aquellos ministerios transitorios; en los ministerios de mediano prestigio encontramos los de agricultura, construcción y trabajo público, educación, ambiente y recursos naturales, salud y seguridad social, industria y comercio, justicia, trabajo, transporte, comunicación y planeamiento o desarrollo. Finalmente, para estas autoras, los gabinetes de alto prestigio son los de finanzas, economía, relaciones exteriores, gobierno e interior, y defensa o seguridad pública.

A su vez, trabajos más recientes establecen que dicha categorización se sustenta sobre los estereotipos de género respecto a los roles sociales que tienen mujeres y varones. En este sentido, los ministerios de mayor jerarquía o alto prestigio se vinculan directamente a actividades concebidas como masculinas. Mientras que los de bajo prestigio sugieren actividades que socialmente se relacionan con la mujer y su femineidad (Krook y O’Brien, 2012; Poskocilova, 2015). Estos trabajos también establecen que, históricamente, las mujeres estuvieron asignadas a un menor número de ministerios, que solían ser caracterizados como de menor prestigio (Davis, 1997; Reynolds, 1999; Russel y Delancey, 2002). En las últimas investigaciones se sostiene que, a pesar del aumento de mujeres en ministerios "masculinos", los patrones de género no cambian drásticamente (Krook y O’Brien, 2012). No obstante, Liu y Banaszak (2016) concluyen que las mujeres que acceden a cargos ministeriales tienen mayor capaci- 
dad política para afectar la formación de política pública, en relación con mujeres en otros espacios de decisión pública; y por ello la importancia de sumarlas a dichos ámbitos.

A continuación, se especifican las características del sistema político de los tres países seleccionados para poder luego observar lo sucedido específicamente en la composición de los ministerios en clave de género.

\section{Argentina}

La República Argentina cuenta con un Poder Ejecutivo nacional que es ejercido por el presidente, un puesto que se renueva cada cuatro años, y un Poder Legislativo, compuesto por la Cámara de Diputados y la Cámara de Senadores (ambas conforman el Congreso Nacional) ${ }^{2}$.

El primer gran paso en cuanto a ejercicio electoral femenino en Argentina se dio en el año 1947, cuando se sancionó la Ley 13.010 que establecía que las mujeres tenían el derecho a participar en elecciones (Barrancos, 2002). Esta ley permitió la inclusión de las mujeres en el sistema político, reconociéndoles uno de los derechos políticos más importantes. A su vez, la reforma constitucional de 1949 legalizó la participación de éstas, que votaron por primera vez en las elecciones nacionales del año 1951. Sin embargo, algunos autores plantean la necesidad de pensar que, aun dándose este paso, siguieron existiendo barreras para la mujer, particularmente la posibilidad de ser elegidas (Caminotti, 2008).

Hoy la mujer representa el 51,17 \% del total de la población argentina ${ }^{3}$, lo que la posiciona como un actor importante y decisorio de la vida social y política. En este sentido, aquella ley de 1947, que permitió la participación femenina en la política, fue el primer paso para la equiparación política entre varones y mujeres. Pero a medida que pasaron los años fue necesario modernizar y ampliar este nuevo ámbito en el que la mujer se desarrolla, no solamente desde el mero ejercicio del voto, sino también desde la posibilidad de proponerse como candidata y resultar electa.

Durante la década de los 90 se dio en Argentina una de las afirmaciones positivas de género más importante: la Ley de Cupo Femenino (Ley Nacional $\left.\mathrm{N}^{\circ} 24.012\right)^{4}$. Fue la primera en América Latina y marcó el punto de inicio

2 El cargo de diputado nacional se ejerce por un periodo de 4 años, la elección de estos se realiza mediante un sistema de representación proporcional con listas partidarias cerradas y bloqueadas; mientras que quien salga electo senador nacional ejercerá por un período de 6 años, y la elección se rige por un sistema de mayoría atenuada que asegura la representación de la segunda fuerza en cantidad de votos.

3 Porcentaje obtenido a partir de información recabada del Censo Poblacional 2010. Sobre un total de 40.091 .359 personas, 20.516 .140 son mujeres.

4 La ley establece un mínimo de $30 \%$ de presencia femenina en las listas de candidaturas a cargos de representación popular; incluye en su texto una cláusula de expectabilidad por la que
De presidentes y ministros. Cuestión de género en los casos de Argentina, Brasil y Chile

María Emilia Perri 
Dossier América Latina: género y política en cuanto a afirmaciones positivas en la región. A ella la acompañó en 1995 la Conferencia de Beijing y la aprobación de su Plataforma de Acción, donde los mecanismos de acción positiva encontrarían una fuerte legitimación a nivel mundial (Caminotti, 2008). Dicha ley fue reformada durante el año 2017. Se sancionó una Ley de Paridad que establece que las listas a cargos de representación nacional deben conformarse en un $50 \%$ por mujeres ${ }^{5}$. Esta situación estuvo dada por los reclamos de las mujeres vinculados a que la ley de cupo se había transformado en un techo difícil de romper y el cual los partidos respetaban como expresión máxima de la participación femenina (Archenti y Tula, 2009).

El impacto de la ley de cuotas en este país es innegable, la representación de las mujeres en el año 1989 (antes de la ley de cupo) era del 8,7 \%, mientras que, en el año 2005, las mujeres representaron el $35 \%$ del Congreso. En Argentina este éxito está relacionado con la compatibilidad con el sistema electoral, dado que el sistema de representación proporcional, sumado a las listas cerradas y bloqueadas fue combinado con un mandato de posición y un alto grado de magnitud partidaria, lo que garantizó un piso mínimo de mujeres electas ${ }^{6}$.

A ello se le sumó la utilización de planes que benefician la igualdad entre varones y mujeres como fue el Plan de Igualdad de Oportunidades 1995-1999 y el Consejo Nacional de la Mujer. Durante los primeros años de retorno a la democracia, se creó la Dirección Nacional de la Mujer que dependía de la Secretaría de Desarrollo Humano y Familia del Ministerio de Salud y Acción Social. Luego la dirección se transformó, en el año 1987, en la Subsecretaría de la Mujer. Ya en el año 1992 se sustituyó la Subsecretaría por el Consejo Nacional de la Mujer (CNM), que dependía de la Presidencia de la Nación. Desde este organismo se buscará la institucionalización del cupo femenino en el país, aprobado un año antes. En la actualidad, se cuenta con el Instituto Nacional de las Mujeres, el cual tiene a su cargo las políticas públicas de igualdad de oportunidades y trato entre varones y mujeres, y forma parte del Ministerio de Salud y Desarrollo Social.

Desde el retorno a la Democracia en el año 1983, de un total de 10 presidentes, solo 1 mujer ha accedido al cargo. Cristina Fernández fue elegida presidente en las elecciones del año 2007, cargo que renovó en las elecciones del 2011.

se establece un mandato de posición. Dado que esto último se prestaba a confusión por la imprecisión del texto, se llevaron a cabo varias presentaciones ante la Justicia (Fallo No 1.513/93; Fallo No 1.565/93; Fallo No 1.602/93; Fallo No 2.931/01), y se dictaron tres decretos reglamentarios posteriores a la ley $(179 / 93,1.246 / 00$ y $451 / 05)$.

5 Durante el año 2019 se llevarán a cabo las primeras elecciones bajo este sistema de paridad.

6 Programa de las Naciones Unidas para el Desarrollo -PNUD (2011); Aportes para el desarrollo humano en Argentina/2011.Género en cifras: mujeres y varones en la sociedad argentina, Buenos Aires, pág. 50. 


\section{Brasil}

En la República Federativa del Brasil el sistema de gobierno también es presidencialista, y el poder legislativo es bicameral. El Poder Ejecutivo nacional es ejercido por el presidente de la República, quien es elegido de forma directa y mediante sufragio universal por un término de cuatro años, pudiendo ser reelegido para un sólo término subsiguiente. Para ser elegido presidente, un candidato debe alcanzar la mayoría absoluta de votos, descontando los votos en blanco y los nulos. En caso de que ese requisito no se cumpla en la primera vuelta, se celebra una segunda vuelta entre los dos postulantes con mayor número de votos, en el que será elegido el que obtenga la mayoría de los votos válidos. El Poder Legislativo brasileño es ejercido por el Congreso Nacional, compuesto por la Cámara de Diputados y el Senado Federal ${ }^{7}$.

La apertura a la vida política de las mujeres en este país se dio en el año 1932, siendo uno de los primeros países latinoamericanos en conceder el voto femenino, aunque esto no significó un aumento en el ingreso de las mujeres a cargos electivos (Marx, Borner y Caminotti, 2007).

Luego del proceso de redemocratización hubo una intensa participación femenina, pero esto no se transformó en logros políticos electorales. Por el contrario, no fue hasta 1997 en que se aprobó la Ley de Cupo Femenino en este país, y aun así los resultados de dicha implementación no fueron los deseados. La ley electoral No 9540 establece la reserva de un porcentaje mínimo y máximo de candidaturas de hombres y mujeres a todos los cargos electos proporcionales. El problema principal que presenta dicha norma es que no prevé ningún sistema de sanción para los incumplimientos de las cuotas, sumado a que cada partido está habilitado a presentar el $150 \%$ de los escaños en juego, lo que lleva a que las mujeres pueden quedar en lugares no expectables de resultar electas (Htun, 2003; Marx, Borner y Caminotti, 2007). Esto produjo que, cuando se implementó por primera vez dicha ley, el número de legisladoras disminuyó (Araújo, 2008a; 2008b).

7 Los legisladores en la Cámara de Diputados (513 miembros) cuentan con un mandato de 4 años, y son elegidos de manera directa por representación proporcional en cada estado y el Distrito Federal. Las elecciones se llevan a cabo bajo el sistema de representación proporcional (RP) por listas abiertas. Cada elector dispone de un sólo voto, que puede emitir a favor de un partido político o de un candidato en lo individual. Los votos que recibe cada candidato de un partido se suman para formar un gran total partidista, el cual es utilizado para determinar el número de escaños que le serán asignados al partido; luego las bancas serán otorgadas a aquellos candidatos con mayor cantidad de votos. La distribución de escaños para la Cámara de Diputados se hace mediante la fórmula D’Hondt. Por su parte, el Senado Federal (81 miembros) es elegido de manera directa para un término de ocho años, pero las elecciones al Senado se celebran cada cuatro años, alternadamente para un tercio (27), o dos tercios (54) de las bancas. Cada estado y el Distrito Federal eligen a tres senadores por el método de mayoría simple, a través del cual resultan electos a los cargos los candidatos con mayor número de votos.
De presidentes y ministros. Cuestión de género en los casos de Argentina, Brasil y Chile

María Emilia Perri 
América Latina: género y política
Por otra parte, también se debe considerar como una apertura de las instancias de poder, la creación del Consejo Nacional de los Derechos de la Mujer (CNDM, vinculado al Ministerio de la Justicia) en el año 1985. Su función era la de formulación, monitoreo y evaluación de políticas públicas. A su vez, Brasil cuenta con las Conferencias Nacionales por la Mujer, que producen el material para la elaboración de los Planes Nacionales de Políticas para las Mujeres ${ }^{8}$.

Actualmente la mujer brasileña representa un $51 \%$ del electorado a nivel nacional. No obstante, estos datos no se traducen en una participación efectiva dentro de los ámbitos de representación política ${ }^{9}$. Siguiendo con este análisis, desde el retorno a la democracia en Brasil solamente una mujer ha accedido al cargo de presidente de la Nación (de un total de 7 presidentes). Dilma Rousseff accedió a dicho cargo a través de elecciones en el año 2011 y fue destituida del mismo por juicio político en el año 2016.

\section{Chile}

La República de Chile posee un sistema presidencialista, en donde la selección del presidente se realiza por votación directa y por mayoría absoluta de los sufragios válidamente emitidos (Artículo 26 de la constitución política), por el término de cuatro años y sin posibilidad de reelección. En el caso de que no haya un candidato que logre obtener más de la mitad de los sufragios (mayoría absoluta), el sistema prevé un sistema de segunda vuelta o "balottage" para las primeras dos posiciones. En cuanto al poder legislativo, este país cuenta con un sistema de doble cámara (Cámara de Diputados y Senado); se lo suele denominar sistema de bicameralismo igualitario dado que no presentan diferencias importantes en cuanto al ejercicio legislativo ${ }^{10}$ (Santibáñez Handschuh et al., 2001).

8 Información recabada del II Plan Nacional de Políticas para las Mujeres, Brasilia 2009.

9 Información obtenida del Instituto Brasilero de Geografía y Estadísticas (IBGE).

${ }^{10}$ Según lo establecido en el artículo 43 de la Constitución Política de la República de Chile y la reforma política realizada en el año 2017, la Cámara de Diputados se compone de 155 miembros electos de manera directa por un término de cuatro años en sesenta distritos de dos miembros cada uno. El Senado cuenta con 50 miembros que son electos de manera directa para un término de ocho años en diecinueve circunscripciones de dos miembros. Las elecciones al Senado se celebran cada cuatro años para aproximadamente la mitad de los escaños; las regiones de número impar escogen a sus senadores en un período, mientras que las regiones de número par y la Región Metropolitana de Santiago celebran elecciones senatoriales en el siguiente período. Al sistema electoral utilizado en las elecciones legislativas en Chile se le conoce como sistema binominal. En principio, se trata de un sistema de representación proporcional -el procedimiento para el reparto de escaños en los distritos y circunscripciones binominales implementa de manera implícita el método de la media mayor (la regla D'Hondt)pero la proporcionalidad se limita a las primeras dos listas, y la lista en segundo puesto puede alcanzar un cincuenta por ciento de la representación (uno de dos escaños) con poco más de un tercio de los votos. 
La inclusión política de la mujer comenzó en el año 1884, momento en el que un número de mujeres se presentó a votar dado que la legislación electoral no les negaba explícitamente el voto, sin embargo, no lo consiguieron. No fue hasta 1934 que consiguieron poder votar en elecciones municipales, y recién en el año 1949 lograron el derecho no sólo de votar, sino también de resultar electas (Ananth, 2009). El caso chileno es un caso estudiado por la no adopción de cuotas electorales, aun siendo un país con una fuerte institucionalización de la lucha por la equidad de género. Durante el período de redemocratización chileno las diferentes asociaciones de mujeres buscaron poner en debate la cuestión de sus derechos de género.

Luego de la restitución de la democracia, en el año 1991, con el fin de promover la igualdad de oportunidades entre varones y mujeres, el gobierno chileno creó, a través de la ley 19.923, el Servicio Nacional de la Mujer (SERNAM). La misión de este organismo es la de "Diseñar, proponer y coordinar políticas, planes, medidas y reformas legales, a través y en conjunto con los distintos ministerios y servicios, conducentes a garantizar y visibilizar la igualdad de derechos y oportunidades entre hombres y mujeres, incorporando en la agenda pública las problemáticas que afectan a la mujer y la familia" ${ }^{\prime 1}$.

El SERNAM comenzó a implementar los llamados Planes de Igualdad de Oportunidades para las Mujeres, que determinaban las prioridades de acción para facilitar el diseño de políticas públicas pro-mujer y que estaban enmarcadas en el contexto de la Cuarta Conferencia de Naciones Unidas sobre la Mujer -Beijing 1995- (Valdés, 2011). Según Franceschet (2008a) este organismo está mejor posicionado que las legisladoras para legislar sobre cuestiones de derechos de las mujeres, dado el fuerte dominio del ejecutivo en el sistema político chileno.

En Chile (hasta el 2017) no hubo ninguna regla institucional que permitiera la mayor participación de las mujeres en ámbitos políticos. Fue una larga discusión pública la inclusión o no de cuotas de género, como medida para aumentar dicha participación:

Tardíamente Chile se incorpora al debate teórico y a la posibilidad de legislar sobre "ley de cuotas" o "paridad". Se presentará en el año 1997 un primer proyecto que "proponía que ninguno de los sexos pudiera superar el $60 \%$ en las listas de candidatos/as para elecciones parlamentarias y municipales como también en los integrantes de los órganos colegiados de los partidos politicos. Este proyecto fue archivado sin discusión, sin apoyo del Ejecutivo, sin urgencia. (Castillo, 2011, p. 35)

Dichos debates finalizarán en el año 2017 con la incorporación de una legislación que establece que no pueden presentarse listas que superen la presencia de un sexo en un $60 \%$. Si el partido no respeta dicha norma, el Servicio

${ }^{11}$ Objetivos establecidos por el SERNAM en su página web http://portal.sernam.cl/. Fecha de acceso: 3 de abril de 2018 .
De presidentes y ministros. Cuestión de género en los casos de Argentina, Brasil y Chile

María Emilia Perri 
Dossier América Latina: género y política

Electoral cuenta con la facultad de rechazar la candidatura de la lista presentada. Es por esto por lo que el empoderamiento de las mujeres chilenas en política se dio a través de la incorporación de más mujeres a las carteras ministeriales, cuestión que se desarrollará en apartados siguientes (Franceschet, 2008b).

En cuanto a la llegada de una mujer al ejecutivo nacional, podemos señalar que desde 1990 (retorno a la democracia chilena) una mujer ha accedido a dicho cargo en dos oportunidades. Michelle Bachelet logró acceder por primera vez a la presidencia en el año 2006, y luego en el año 2014 fue electa nuevamente para el cargo.

A continuación, y a partir de lo hasta aquí expuesto, se presenta el análisis en torno al vínculo entre presidentes y ministros bajo perspectiva de género. ¿Impacta el sexo del presidente a la hora de la conformación de su gabinete ministerial?

\section{Poder Ejecutivo ¿Espacio femenino?}

La inclusión de la cuestión de género dentro de los ejecutivos se ha dado en primer lugar a través de direcciones que promovían específicamente el rol de la mujer en la sociedad. Quizás el caso más paradigmático es el de Chile, por el papel que cumplió el SERNAM y por la capacidad que adquirió a medida que avanzaban los años. En Brasil y Argentina las Direcciones por las mujeres no han tenido este rol tan relevante políticamente.

Pero para poder ver claramente que sucede con las mujeres en los ejecutivos, debemos observar en primer lugar las carteras ministeriales, dado que este es uno de los lugares de mayor empoderamiento. Según estudios realizados por IDEA (2008), en noviembre de 2007 Chile tenía un porcentaje de mujeres en gabinete del 36,4 \% (siendo el segundo país en América Latina con el porcentaje más alto de mujeres ministras), Argentina el $25 \%$ y Brasil el 14,3\%.

Cuando se analiza según la división de ministerios de Escobar-Lemmon y Taylor-Robinson (2009), podemos ver como las mujeres están sobrerrepresentadas en gabinetes ministeriales de mediano prestigio, mientras que en los de mayor prestigio dicho género se encuentra subrepresentado.

En el caso de Argentina, el mayor número de mujeres ministras se concentra en los ministerios denominados de mediano prestigio, y será durante las dos presidencias de Cristina Fernández. Se observa un incremento de mujeres a la cabeza de los ministerios de mayor prestigio $^{12}$, que luego se sostiene durante la presidencia de Mauricio Macri. A pesar de ello, el número de mujeres no logra superar el $20 \%$ del total de ministros en todo el periodo. De un total de 283 personas, solo 28 mujeres accedieron a cargos de ministras, traduciéndose en un $9,89 \%$.

\footnotetext{
${ }^{12}$ Ejemplo de esto será el caso de Nilda Garré, que logró ser ministra de defensa, y luego fue designada ministra del nuevo Ministerio de Seguridad.
} 
Lo interesante en el caso argentino es que, salvo en la última presidencia de Fernández, no hay mujeres en ministerios denominados de bajo prestigio. Por el contrario, a lo largo del periodo se posicionan en los ministerios de mediano y de alto prestigio. La mujer comienza a aparecer en estos cargos a partir del año 1995, con la segunda presidencia de Menem. Esto podría vincularse a que comienzan a visibilizarse las mujeres en política a partir de la ley de cupo, implicándose de esta manera en el armado político y logrando así la postulación para los ministerios. Previo a este periodo solo encontramos una mujer, Susana Ruiz Cerruti, como ministra de relaciones exteriores en el año 1989.

Durante la presidencia de Menem encontramos mujeres en ministerios de educación. Mientras que durante las presidencias dadas entre los años 2000 y 2003 encontramos ministras dentro de las carteras de trabajo, seguridad social y educación. Con los gobiernos kirchneristas (2003-2015, tres mandatos) comienzan a posicionarse mujeres en ministerios de alto prestigio o vinculados a actividades masculinas: defensa, seguridad, economía; y siguen posicionándose en salud, desarrollo social y producción.

El principal problema observado es que el gobierno de Macri (2016-2018) corta con una escalada femenina en las carteras ministeriales, bajando su presencia de un $18 \%$ a un $9 \%$. Esto debe analizarse a la luz de que, dentro de Argentina, en los últimos años, se ha dado un movimiento de mujeres muy fuerte que busca ampliar los horizontes de participación y de decisión femeninos, lo cual al observar estos datos no ha logrado impactar en la construcción de carteras ministeriales femeninas dentro del gobierno. A pesar de ello, el número de mujeres aumenta en ministerios de alto prestigio, en relación con la presencia de sus pares varones.

En Brasil las mujeres se nuclean en carteras ministeriales de mediano y bajo prestigio, sumado a que a medida que pasan los años pierden también los pocos ministerios de alto prestigio que tenían.

Al analizar el período en su conjunto, vemos que desde el retorno a la democracia y durante treinta años aumenta progresivamente el porcentaje de ministras. Es importante tener en cuenta que el incremento de mujeres, que logra superar el $20 \%$, se da con la llegada de la presidenta Dilma Rousseff. Esta mandataria logra aumentar sustantivamente el número de mujeres ministras, sin embargo, no llega a romper con la constante de que las mujeres no alcanzan carteras ministeriales de alto prestigio.

En términos generales, las mujeres representan el 7,93 \% de los ministerios durante todo el período analizado, sobre un total de 529 ministros. Durante la presidencia de Fernando Collor de Mello y de Itamar Franco, las mujeres se ubican en ministerios de Finanza, Hacienda y Trabajo. Con la llegada de Fernando Henrique Cardoso la situación no se modifica profundamente, dado que las mujeres ministras se encuentran en ministerios de Integración, Turismo o Desarrollo.

Bajo las presidencias de Luiz Inácio Lula da Silva y Dilma Rousseff, el número de mujeres aumenta, pero siguen siendo, como se dijo previamente, ministerios de mediano y bajo prestigio. Ejemplo de ello serán los ministerios
De presidentes y ministros. Cuestión de género en los casos de Argentina, Brasil y Chile

María Emilia Perri 
Dossier América Latina: género y política de Cultura, Desarrollo Social, Medio Ambiente, Energía o Comunicación. Finalmente, en el período analizado, el último presidente (Michel Temer) no tuvo mujeres dentro de su gabinete. Cuestión que podría ser pensada como un retroceso para la participación femenina en política, ya que no promueve instancias de apertura a las mujeres en la cúpula del poder ejecutivo.

En Chile, a medida que van transcurriendo los años se va dando un incremento de mujeres en los ministerios, sumado con una apertura de los de alto prestigio para las mujeres. En este país se ve un amplio número de mujeres ministras gracias a que luego de la llegada a la democracia varios gobiernos buscaron equiparar, en términos de género, las direcciones de los ministerios. En todo el periodo, sobre un total de 363 ministros, las mujeres logran acceder a un $25 \%$ de cargos.

Durante la presidencia de Eduardo Frei Ruiz-Tagle las mujeres se ubican en los ministerios de Justicia y Bienes Nacionales. Mientras que Ricardo Lagos Escobar incorporó mujeres en los ministerios de Salud, Defensa, Relaciones Exteriores, Planificación, Educación, Vivienda y Bienes Nacionales, lo cual muestra una ampliación de espacios hacia las mujeres.

En el año 2006, Michelle Bachelet accede por primera vez a la presidencia. Es así como, llevando adelante una promesa de campaña, la de la conformación de su gabinete ministerial de forma paritaria, Bachelet designó a sus ministros, teniendo en cuenta lo antes dicho y buscando que haya igualdad de género en el número. Esto implica que en los dos períodos que comprende su presidencia, el número de mujeres supere el $35 \%$. Las mujeres durante sus gobiernos accedieron a los ministerios de Trabajo, Economía, Gobierno, Salud, Defensa, Minería, Planificación, Agricultura, Educación, Vivienda, Bienes Nacionales y Cultura.

Las gobernaciones de Bachelet se intercalan con los gobiernos de Sebastián Piñera (2010 a 2014 y desde 2018 a la fecha). Dicho presidente posicionó a las mujeres en Vivienda, Bienes Nacionales, Trabajo, Gobierno y Medio Ambiente.

Con la segunda presidencia de Piñera, nuevamente las mujeres pierden el acceso a los ministerios de mayor prestigio y aumentan exponencialmente su incorporación a ministerios de bajo prestigio. Si a esta cuestión le sumamos que, en su primera presidencia, el número de participación femenina decreció un $25 \%$, estaríamos en condiciones de decir que el presidente Piñera no se caracteriza por pensar en la incorporación femenina dentro del poder ejecutivo, por lo menos no a la cabeza de las carteras ministeriales.

Al observar lo sucedido en los tres países, se puede concluir que la importancia que tiene la inclusión de mujeres en cargos ejecutivos está dada sobre todo en los países donde la ley de cupo no logra implementarse o tener éxito, por lo que la inclusión en el ejecutivo de la cuestión de género se transforma en la puerta que tienen las mujeres para entrar en política. El ejemplo de Bachelet da cuenta de esto, dado que ella ganó notoriedad pública en los cargos de Ministra de Salud (2000-2002) y de Ministra de Defensa (2002-2004). 
Otro caso de este estilo fue el de Dilma Rousseff, quien se desempeñó como Secretaria de Energía (1991-1995) y luego como Ministra de Energía (2003-2005), ganando un lugar en el ojo público que le permitiría luego ser la candidata a las elecciones presidenciales.

Por el contrario, en el caso de Argentina, Cristina Fernández llegó a la presidencia mediante una vasta carrera legislativa, primero como diputada provincial (1989-1993), luego intercalando períodos como senadora nacional (1995-1997 y 2001-2005) y como diputada nacional (1997-2001). Esta cronología permite ver cómo ella logró su carrera nacional en el marco de la implementación de la ley de cupo femenino argentina.

Al pensar los tres países en su conjunto, podemos establecer un vínculo entre la presencia de una presidenta mujer y el aumento de ministras. El problema observado en este análisis es la incapacidad de éstas de generar conciencia en torno a su rol dentro del ejecutivo, lo cual se demuestra que ante la aparición de un presidente varón, se pierde el número de ministras, teniendo casos emblemáticos como los de la presidencia de Piñera en Chile, Temer en Brasil o Macri en Argentina.

\section{Consideraciones finales}

En este trabajo se buscó hacer una comparación entre tres países que, compartiendo grandes similitudes políticas, sociales y culturales, presentan diversas realidades en cuanto a mujer y participación política. El análisis estuvo direccionado a entender la relación entre presidentes y ministros, siendo la pregunta guía si la aparición de mujeres presidentas genera mayor apertura de cargos ministeriales para el colectivo femenino.

En cuanto a los sistemas electorales, los tres países presentan diferencias muy visibles que generaron la integración de sus mujeres de maneras diferentes. Argentina con una robusta ley de cupo que planteó el ámbito legislativo como primera puerta de ingreso al mundo político (ejemplo de ello será la carrera política de Cristina Fernández). Por su parte, en Brasil las mujeres tuvieron que luchar por los espacios y encontrar salida a una mala legislación de cuotas; Dilma Rousseff encontrará su espacio en secretarías y ministerios y desde allí construirá su capacidad política. Por último, al no contar Chile con una legislación que les generará apertura de espacios políticos, será el SERNAM el lugar para gestionar la participación de mujeres; Michelle Bachelet, al igual que Rousseff encontrará su espacio dentro del ejecutivo, construyendo su carrera política en torno al rol de ministra.

La hipótesis planteada en la introducción fue corroborada por los datos cuantitativos presentados. El que hubiera mujeres a la cabeza del ejecutivo nacional se tradujo en aumento de ministras. El estudio de los tres casos latinoamericanos permite ver cómo, a medida que llegan mujeres al cargo de presidente, aumenta sustancialmente el número de ministras. Sin embargo, no
De presidentes y ministros. Cuestión de género en los casos de Argentina, Brasil y Chile

María Emilia Perri 
Dossier América Latina: género y política así en aquellos ministerios que permiten un mayor control de políticas (ministerio de alto prestigio), lo que permite pensar que las mujeres presidentas no logran romper con las lógicas socioculturales de dominio masculino y los estereotipos de género respecto a los roles sociales de varones y mujeres (Krook y O’Brien, 2012). El vínculo entre mujer presidenta y carteras ministeriales femeninas de alto prestigio solo se puede observar en Argentina.

Estos números nos permiten pensar en la configuración del poder hacia dentro de los poderes públicos. La realidad nos dice que, a pesar de tener mujeres en los legislativos o ejecutivos y de incluso haber logrado posicionar mujeres como presidentas, la perdida de estas últimas se traduce en un retroceso en el resto de los espacios. Para ello alcanza con solo mirar Brasil o Chile en los últimos años. Las mujeres se desdibujan en los ministerios ante la llegada de un presidente masculino.

A su vez, las discusiones en torno al lugar de la mujer en lo social y a nivel mundial parecen no impactar en los últimos gobiernos de los tres países. Los ministerios que ocupan las mujeres siguen reproduciendo la división sexual del trabajo: las mujeres en ministerios o secretarías vinculadas a niñez, familia, cuidado, desarrollo social, salud o educación y los varones a defensa, economía, obra pública o relaciones internacionales (Poskocilova, 2015).

Se hace necesario pensar otras lógicas para modificar las estructuras de poder. No es suficiente lo numérico, hay que adentrarse en los partidos políticos, en las formas de trabajo que plantean las estructuras públicas. Será esto lo que permita transformar la cultura política de los países y con ello asegurar la manera de vincularse equitativamente al poder.

El problema que se suscita, y que ha quedado fuera del análisis aquí realizado, es justamente cómo son las carreras políticas de las mujeres, qué es lo que pasa con la inclusión de ellas en los partidos políticos, dado que son estos los que luego las postulan a los cargos. La realidad es que diferentes estudios muestran que el problema principal de las políticas es la apertura partidaria.

Las mujeres en Latinoamérica han recorrido un largo camino en cuanto a igualdad de género, y eso lo demuestran los tres países aquí estudiados. Sin embargo, todavía queda mucho por recorrer, pues las cúpulas partidarias siguen siendo lugares masculinos, con lógicas masculinas, que deben modificarse para poder pensar una verdadera y completa inclusión política de mujeres.

Finalmente, y pensando el título del trabajo, existe un vínculo entre los cargos políticos (presidentes y ministros) y la ampliación de espacios para la mujer. Es necesario decir que la aparición de mujeres ministras no es solo una ampliación de derecho, sino que para algunos casos es la manera que tienen las mujeres de jugar el juego político, y a partir de allí pensar su carrera política. Pero, por otro lado, el no planteo de manera sistemática de pensar ministerios paritarios muestra como todavía se soslaya la cuestión de la mujer en política y de los inconvenientes que tiene para hacerlo, y por ello entonces podría traducirse en restricción de derecho. 
El presente trabajo buscó señalar algunos horizontes para pensar cómo se dan las estructuras ejecutivas de poder y el acceso de mujeres al mismo desde una perspectiva de género. Los desafíos para el futuro están vinculados a lo antes mencionado: lograr incorporar la mirada de género en ámbitos políticos que hoy reproducen una configuración masculina del poder, y que impacta en la manera en que las mujeres logran incorporarse en política. Esto será lo que la democracia del siglo XXI deberá trabajar en términos de igualdad política.

\section{Referencias bibliográficas}

Ananth, A. (2009). Beyond the Ley de Cupos. The Variation in Gender Empowerment between Argentina and Chile. CUREJ - College Undergraduate Research Electronic Journal. University of Pennsylvania.

Araújo, C. (2008). ¿Por qué las cuotas no funcionan en Brasil? En M. Ríos Tobar, Mujer y Política. El impacto de las cuotas de género en América Latina. Santiago de Chile: IDEA Internacional, FLACSO-Chile y Editorial Catalonia.

Araújo, C. (2008). Mujeres y elecciones legislativas en Brasil: las cuotas y su (in)eficacia. En N. Archenti y M. I. Tula, Mujeres y política en América Latina. Sistemas electorales $y$ cuotas de género. Buenos Aires: Editorial Heliasta.

Archenti, N. y Tula, M. I. (2008). Mujeres y política en América Latina. Sistemas electorales $y$ cuotas de género. Buenos Aires: Editorial Heliasta.

Archenti, N. y Tula, M. I. (2009). Representación política, sistemas electorales y género. Análisis de las listas partidarias en cinco distritos subnacionales, Argentina 2007. En IX Congreso Nacional de Ciencia Política. Organizado por la Sociedad Argentina de Análisis Político y la Universidad Nacional del Litoral. Santa Fe.

Archenti, N. y Tula, M. I. (2014). La representación política imperfecta. Logros y desafíos de las mujeres politicas. Buenos Aires: Editorial EUDEBA.

Baldez, L. (2012). Gender. En P. Kingstone y D. Yashar, Routledge Handbook of Latin American Politics (pp. 319-332). New York: Routledge.

Barrancos, D. (2002). Inclusión/Exclusión. Historia con Mujeres. Buenos Aires: Fondo de Cultura Económica.

Borrelli, M.A. (2002). The President's Cabinet: Gender, Power, and Representation. Boulder: Editorial Lynne Rienner.

Caminotti, M. (2008). Derribar los muros indebidos: Reflexiones en torno de las leyes de cupo femenino en argentina. Revista Aportes para el Estado y la Administración Gubernamental, año 14, no. 25, Buenos Aires.

Castillo, A. (2011). Democracia, políticas de la presencia y paridad. Estudio sobreparticipación política de mujeres en el Ejecutivo (2006-2010). Serie Género y Política, Santiago de Chile: Corporación Humanas.

Davis, R. (1997). Women and Power in Parliamentary Democracies. Cabinet Appointments in Western Europe, 1968-1992. Lincoln: University of Nebraska Press.
De presidentes y ministros. Cuestión de género en los casos de Argentina, Brasil y Chile

María Emilia Perri 
Dossier América Latina: género y política
Escobar-Lemmon, M. y Taylor-Robinson, M. (2005). Women Ministers in Latin American Government: When, Where, and Why?. American Journal of Political Science, vol. 49, no. 4, pp. 829-844.

Escobar-Lemmon, M. y Taylor-Robinson, M. (2009). Getting to the Top. Career Paths of Women in Latin American Cabinets. Political Research Quarterly, vol. 62, no. 4, pp. 685-699.

Escobar-Lemmon, M., Schwindt-Bayer, L. y Taylor-Robinson, M. (2012). Representing women: Empirical insights from legislatures and cabinets in Latin America. Ponencia presentada en el IV Congreso Uruguayo de Ciencia Política, Montevideo, 14-16 noviembre.

Franceschet, S. (2008a). ¿Promueven las cuotas de género los intereses de las mujeres? El impacto de las cuotas en la representación sustantiva de las mujeres. En M. Ríos Tobar, Mujer y Política. El impacto de las cuotas de género en América Latina; Santiago de Chile: IDEA Internacional, FLACSO-Chile y Editorial Catalonia.

Franceschet, S. (2008b). La representación política de las mujeres en un país sin ley de cuotas: El caso de Chile. En N. Archenti y M. I. Tula, Mujeres y política en América Latina. Sistemas electorales y cuotas de género, Buenos Aires: Editorial Heliasta.

Santibáñez Handschuh et al. (2001). El régimen electoral y de partidos políticos en Chile y sus efectos en el sistema político nacional y regional. Revista de Administração Pública, 35(3) Maio/Jun., pp. 197-225, Rio de Janeiro.

Htun, M. N. (2003). Dimensiones de la inclusión y exclusión política en Brasil: Género y raza. Serie de informes técnicos del Departamento de Desarrollo Sostenible, Washington, D.C.: Banco Interamericano de Desarrollo.

Htun, M. N. (2005). Women, Political parties and electoral systems in Latin America. En J. Ballington y A. Karam, Women in Parliament: Beyond numbers (pp. 112-121). Stockholm: International Institute for Democracy and Electoral Assistance (IDEA).

Krook, M. y O'Brien, D. (2012). All the president's men? The appointment of female Cabinet Ministers worldwide. The Journal of Politics, no. 47(3), pp. 840-855.

Llanos, B. y Sample, K. (2008). 30 años de democracia: ¿En la cresta de la ola? Participación política de la mujer en América Latina, Perú: IDEA Internacional.

LIDERA y ELA (2011). La participación de las mujeres en las legislaturas argentina: ¿una cuenta saldada?

Liu, S.S. y Banaszak, L.A. (2016). Do Government Positions Held by Women Matter? A Cross-National Examination of Female Ministers' Impact on Women's Political Participation. Politics \& Gender, vol. 13, Issue 1, March, pp. 132-162.

Marx, J., Borner, J. y Caminotti, M. (2007). Las Legisladoras. Cupos de género y política en Argentina y Brasil. Buenos Aires: Siglo XXI.

Pegoraro, M. y Zulcovsky, F. (2008). Gobierno. En L. Aznar, M. de Luca, Política. Cuestiones $y$ problemas, Buenos Aires: Ariel. 1 Edición.

Poskočilova, P. (2015). Women in executive positions. The influence of institutional factors and gender stereotypes on women's representation in the Peruvian presidential cabinet. Pensamiento Americano, no. 8(14), pp. 92-108.

Programa de las Naciones Unidas para el Desarrollo-PNUD (2011). Aportes para el desarrollo humano en Argentina/2011.Género en cifras: mujeres y varones en la sociedad argentina, Buenos Aires. 
Reynolds, A. (1999). Women in the Legislatures and Executives of the World. World Politics, no. 51(4), pp. 547-73.

Ruiz Seisdedos, S. y Grande Gascón, S. (2015). Participación política y liderazgo de género: Las presidentas latinoamericanas. América Latina Hoy, Ediciones Universidad de Salamanca, no. 71, pp. 151-170.

Russell, C.A y Delancey, M.W. (2002). African Women in Cabinet Positions - Too Few, Too Weak. Asian Women, no. 15(12), pp. 147-63.

Valdés, T. (2011). Acción política de mujeres: 1990-2009. Institucionalizando la equidad de género. En E. Largo (ed.), Autonomía política de las mujeres. Algunas reflexiones, Serie Género y Política, pp. 43-84, Santiago de Chile: Corporación Humanas.
De presidentes y ministros. Cuestión de género en los casos de Argentina,

\section{Brasil y Chile}

María Emilia Perri 